\title{
Quantum-mechanical treatment of cerous magnesium nitrate in an external magnetic field
}

\author{
A. K. Theophilou \\ Nuclear Research Center "Demokritos," Aghia Paraskevi, Attikis, Greece \\ S. Thanos \\ Theoretical and Physical Chemistry Institute, National Hellenic Research Foundation, Vas. Constantinou 48 Ave., \\ Athens 501/1, Greece \\ (Received 31 October 1983)
}

\begin{abstract}
The ground state of cerous magnesium nitrate $\left[\mathrm{Ce}_{2} \mathrm{Mg}_{3}\left(\mathrm{NO}_{3}\right)_{12} \cdot 24 \mathrm{H}_{2} \mathrm{O}\right]$ in a homogeneous external magnetic field is treated quantum mechanically. The results obtained for the critical magnetic field and the magnetization agree with the existing experimental data. However, a serious deviation from the results of the semiclassical treatment appears when an external field is present. This deviation is not only quantitative but also qualitative.
\end{abstract}

\section{INTRODUCTION}

In this paper the quantum-mechanical treatment of the ground state of crystals with dipole-dipole and exchange interactions in an external magnetic field is presented. The classical case was treated in an earlier paper by the same authors. ${ }^{1}$

The interest in studying these crystals is due to the excellent magnetic properties at very low temperatures because their phase transition is in the millikelvin region. $^{2-4}$ In such crystals the exchange interaction is comparable to the dipolar interaction or it is absent. ${ }^{5}$ So, they can be used as low-temperature thermometers. Lower temperatures, above their transition point, can be obtained by the method of adiabatic demagnetization. $\mathrm{Ce}_{2} \mathrm{Mg}_{3}\left(\mathrm{NO}_{3}\right)_{12} \cdot 24 \mathrm{H}_{2} \mathrm{O}(\mathrm{CMN})$ is such a crystal whose transition temperature is $1.8 \mathrm{mK}{ }^{2}$ The exchange interaction seems to be absent in CMN.

Crystals with no exchange interaction are of special theoretical interest (as their Hamiltonian is exact), because no phenomenological terms or parameters are present, contrary to the crystals where the exchange interaction is important. Thus one can directly compare theoretical and experimental conclusions about many-spin systems. However, as the many-spin systems are not exactly solvable, one has to resort to approximation methods. The comparison of theoretical results with experiment is the best way to check the validity of the approximation method.

The ground state of such crystals in the absence of an external magnetic field was extensively studied. ${ }^{6,7} \mathrm{CMN}$ particularly has been studied by Niemeijer and his coworkers. ${ }^{8-10}$

A detailed account of theoretical and experimental results is given in Ref. 6.

Until now, no quantum-mechanical treatment of dipolar crystals in an external magnetic field has been presented. All previous papers are based on the semiclassical theory of Niemeijer ${ }^{5}$ and the theory of Luttinger and Tisza. $^{11}$ One of us ${ }^{12}$ has shown that for Bravais lattices with one ion per unit cell, using one Lagrange multiplier, one can solve the semiclassical problem but only in the case of an external magnetic field normal to the spin orientation in the zero-field ground-state configuration. The authors, in a previous work, show that two Lagrange multipliers are sufficient for solving the problem for any direction of the field. ${ }^{1}$

Theoretical calculations have also been made by Marrenga and Niemeijer ${ }^{13}$ who used the method developed by Theophilou ${ }^{12}$ for the $y$ direction of CMN; for other directions Marrenga and Niemeijer tried various spin configurations to find a lower and an upper bound for the ground-state energy. Broughton and Mullin ${ }^{14}$ employed a Bloch transformation for dealing with the problem.

Since there is so much theoretical work with the semiclassical treatment, it is of interest to explore the problem quantum mechanically and to compare the results with those of the semiclassical treatment. As can be seen from Sec. III, the quantum and semiclassical results do not agree, except in the case of zero external field.

In Sec. II of this paper we treat the Hamiltonian of the cluster of system of ions in an external magnetic field and we develop the method of solution. In Sec. III we present the general conclusions and we compare the semiclassical, quantum-mechanical, and experimental results.

\section{THE DIPOLAR HAMILTONIAN IN AN EXTERNAL MAGNETIC FIELD}

The general form of the Hamiltonian of a system of ions with dipole-dipole and exchange interactions in an external magnetic field is ${ }^{1,5-7}$

$$
\begin{aligned}
\mathscr{H}= & \frac{1}{2} \sum_{\substack{i, j \\
i \neq j}} \frac{1}{R_{i j}^{3}}\left[\vec{\mu}_{i} \cdot \vec{\mu}_{j}-3 \frac{\left(\vec{\mu}_{i} \cdot \overrightarrow{\mathbf{R}}_{i j}\right)\left(\vec{\mu}_{j} \cdot \overrightarrow{\mathbf{R}}_{i j}\right)}{R_{i j}^{2}}\right] \\
& +\sum_{\substack{i, j \\
i \neq j}} v \Delta_{i j} \overrightarrow{\mathbf{S}}_{i} \cdot \overrightarrow{\mathbf{S}}_{j}-\sum_{i} \vec{\mu}_{i} \cdot \overrightarrow{\mathrm{h}}, \\
\Delta_{i j}= & {\left[\begin{array}{ll}
1 & \text { for } i, j \text { nearest neighbors } \\
0 & \text { otherwise },
\end{array}\right.}
\end{aligned}
$$


where the first term is the dipole-dipole interaction, the second term is the exchange interaction, and the third term is the interaction with the external magnetic field; $v$ is the strength of the exchange interaction. For convenience we have used the site vector $\overrightarrow{\mathbf{R}}_{i}$ for denoting the lattice site $i$. The first and the second terms consist of the Hamiltonian $\mathscr{H}$, i.e., the Hamiltonian without an external field. In the case of $\mathrm{CMN}$, the exchange interaction strength $v$ is taken as zero, ${ }^{6}$ but in general, a nonzero value of $v$ does not alter the general validity of the present method.

After applying the procedure followed in Ref. 6, which is based on the Luttinger-Tisza-Niemeijer approximation, ${ }^{4,11}$ the resulting Hamiltonian is
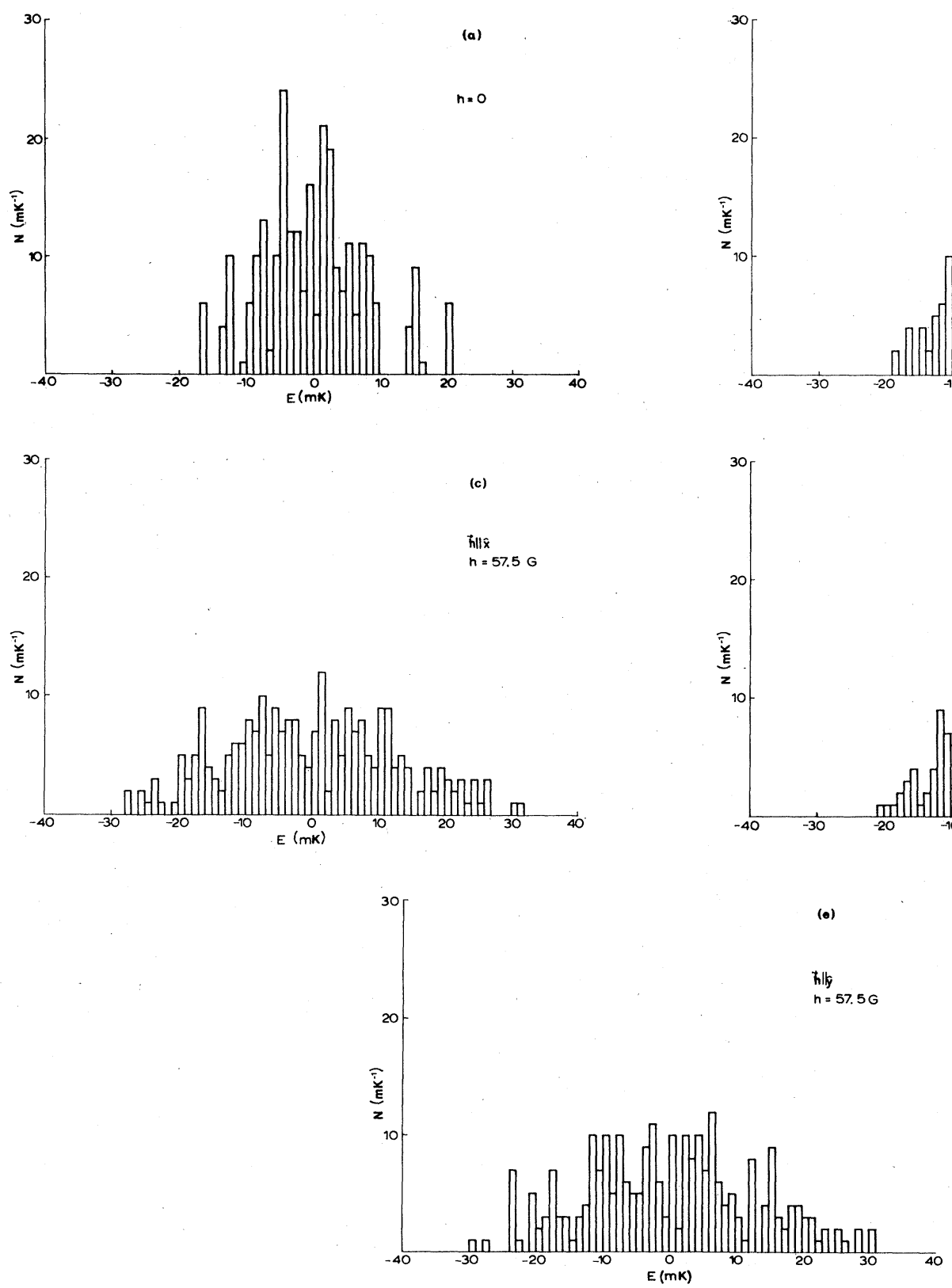

FIG. 1. Density of states versus energy for different values of magnetic field and for different $x, y$ directions of the field. (a), $h=0$; (b)-(e), $h \neq 0$.

$$
\begin{aligned}
H= & \frac{1}{2} \sum_{\substack{\overrightarrow{\mathrm{R}}, \overrightarrow{\mathrm{R}}, \alpha, \beta=x, y, z}} A_{\overrightarrow{\mathrm{R}}, \overrightarrow{\mathrm{R}}}^{\alpha \beta}, S_{\overrightarrow{\mathrm{R}}}^{\alpha} S_{\overrightarrow{\mathrm{R}}}^{\beta}, \\
& +\frac{1}{2} \sum_{\substack{\overrightarrow{\mathrm{R}}, \alpha, \beta=x, y, z}} A_{\overrightarrow{\mathrm{R}}, \overrightarrow{\mathrm{R}}}^{\alpha \beta}\left\langle\Psi\left|S_{\overrightarrow{\mathrm{R}}}^{\alpha}\right| \Psi\right\rangle S_{\overrightarrow{\mathrm{R}}}^{\beta}-\sum_{\overrightarrow{\mathrm{R}}} \overrightarrow{\mathrm{h}} \cdot \vec{\mu}_{\overrightarrow{\mathrm{R}}},
\end{aligned}
$$

where the quantities $A_{R, R}^{\alpha \beta}$ are defined in Refs. 1 and 6 . $|\Psi\rangle$ is the wave function of the crystal. ${ }^{6}$

The initial Hamiltonian, Eq. (1), has the same translational symmetry as the zero-field Hamiltonian, because the external magnetic field is homogeneous. Also the Hamiltonian resulting after the simplification, Eq. (2), has
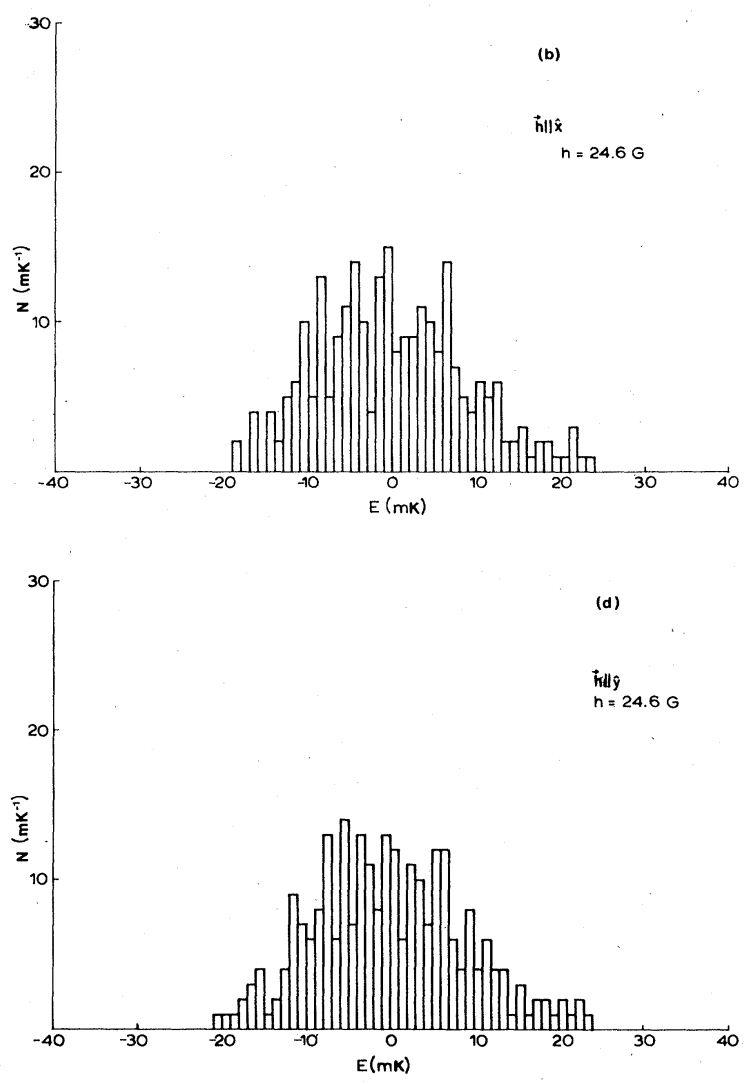

(e) 
solutions belonging to the same irreducible representation of the translation group, as that with the zero-magnetic field. ${ }^{6}$ However, one should note that the point-symmetry operations of the crystal lattice do not leave either Hamiltonian invariant because of the existence of the external magnetic field term. This term

$$
H_{m}=-m_{B} \sum_{\substack{\overrightarrow{\mathrm{R}} \\ \alpha, \beta=x, y, z}} g_{\alpha \beta} h^{\alpha} S_{\overrightarrow{\mathrm{R}}}^{\beta}
$$

can be written as

$$
H_{m}=-\sum_{\beta=x, y, z} h^{\beta} \sum_{\overrightarrow{\mathrm{R}}} S_{\overrightarrow{\mathrm{R}}}^{\beta},
$$

where

$$
h^{\beta}=m_{B} \sum_{\alpha=x, y, z} g_{\alpha \beta} h^{\alpha} .
$$

Since the nonlinear term in the Hamiltonian (2) does not involve the external magnetic field it can be treated as in Ref. 6, i.e., by using a perturbation theory to the correction term

$$
H_{c}=\frac{1}{2} \sum_{\alpha, \beta=x, y, z} \sum_{\overrightarrow{\mathrm{R}}} A_{\overrightarrow{\mathrm{R}}, \overrightarrow{\mathrm{R}}}^{\alpha \beta}\left\langle\Psi\left|S_{\overrightarrow{\mathrm{R}}}^{\alpha}\right| \Psi\right\rangle S_{\overrightarrow{\mathrm{R}}}^{\beta}
$$

with unperturbed operator

$$
H^{\prime}=H-H_{c} \text {. }
$$

The expression of $H^{\prime}$, in terms of the operators $b_{\overrightarrow{\mathrm{k}}}^{\alpha}=\sum_{\overrightarrow{\mathrm{R}}} e^{i \overrightarrow{\mathrm{k}} \overrightarrow{\mathrm{R}}} S_{\overrightarrow{\mathrm{R}}}^{\alpha}$, is given by Eq. (12b) in Ref. 6 to which one must add the magnetic field term

$$
H_{m}=-\sum_{\beta=x, y, z} h^{\beta} b_{0}^{\beta} \text {. }
$$

\section{APPLICATION TO CMN}

In dealing with the magnetic properties of $\mathrm{CMN}$ one has to consider only the $\mathrm{Ce}^{3+}$. The sites of these ions form a primitive rhombohedral lattice with hexagonal pseudounit cell of dimensions $\alpha=11.004 \AA, c=17.296 \AA$, and point-group symmetry $D_{3 d} \cdot 5,15$

All input data for our calculations were the same as those of the zero-field case. ${ }^{7}$ Calculations were done for the field along the $x$ and $y$ directions of the crystal axis (see Ref. 1) for fields ranging between 0 and $100 \mathrm{G}$, i.e., above the critical field beyond which the ground state of the crystal goes to the metamagnetic state..$^{2,4}$

As the eigenstates of our Hamiltonian are too numerous, $2^{8}$ (as explained in Refs. 1 and 6), no details of the states are presented in the paper but instead the density of states is given for various values of the external fields [see Figs. 1(a)-1(e)].

The ground-state energy versus the external magnetic field is given in Fig. 2. As one can see from this figure the ground-state energy of the crystal with the field along the $y$ direction always lies below that for which the field is along the $x$ direction. The two curves coincide for small values of the magnetic field. A well-defined critical field appears for the $x$ direction at $h_{c}=57.5 \mathrm{G}$. This value is very near to the measured experimental value of

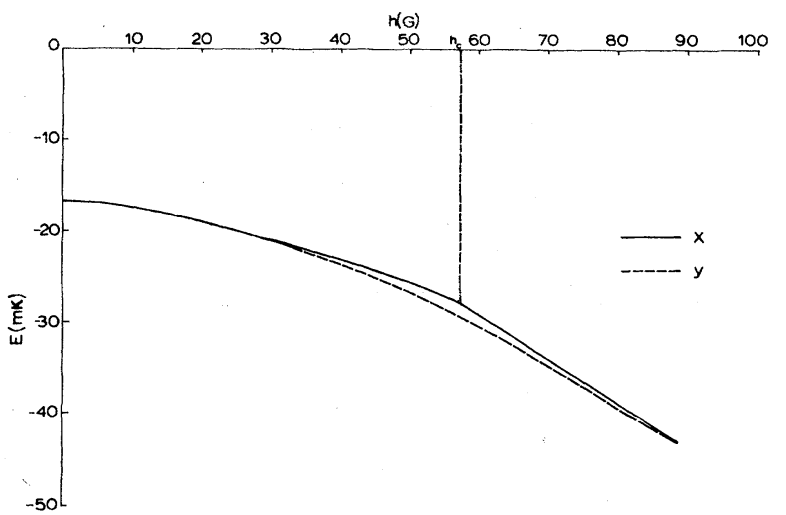

FIG. 2. Ground-state energy versus magnetic field. The solid line and the dashed lines denote the cases when the field is parallel to the $x$ and to the $y$ axis, respectively. The value of the critical field is $h_{c}=57.5 \mathrm{G}$.

$60 \mathrm{G}$ by Fisher, Hornung, Brodale, and Giaugue. ${ }^{2}$

The small deviation of our results from the experimental value is due to our approximation method and possibly to our data for the unit cell. ${ }^{5,15}$ For this case we are planning to perform additional calculations to establish to which extent the critical field is sensitive to the crystal parameters. In this way one will be able to estimate the accuracy of our approximation method. The minima and maxima of energy for the various $k$ states appear in Figs. 3 and 4. It is clear that the lowest energy is that of a $\overrightarrow{\mathrm{k}}=0$ state, with an intersection at zero field with the $\overrightarrow{\mathrm{k}}_{1}, \overrightarrow{\mathrm{k}}_{2}, \overrightarrow{\mathrm{k}}_{3}$ states. This degeneracy was discussed in a previous paper. ${ }^{7}$ The definition of $k$ vectors is explained in Refs. 1 and 6.

It is interesting to note that for the field along the $y$ direction no clear transition field appears, but a gradual changeover toward the metamagnetic state appears. This is also the case in the semiclassical calculation, although

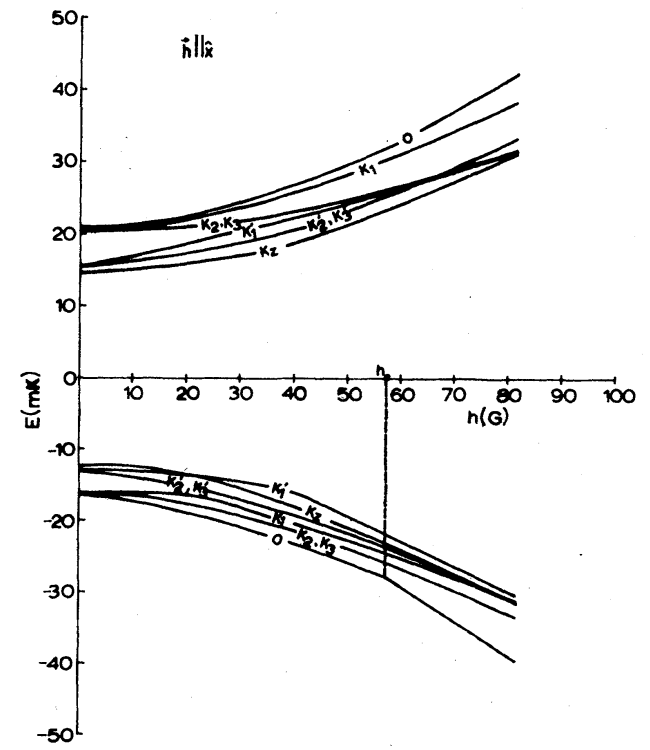

FIG. 3. Maximum and minimum energy for any $k$ representation versus magnetic field when the field is parallel to $x$ axis. 


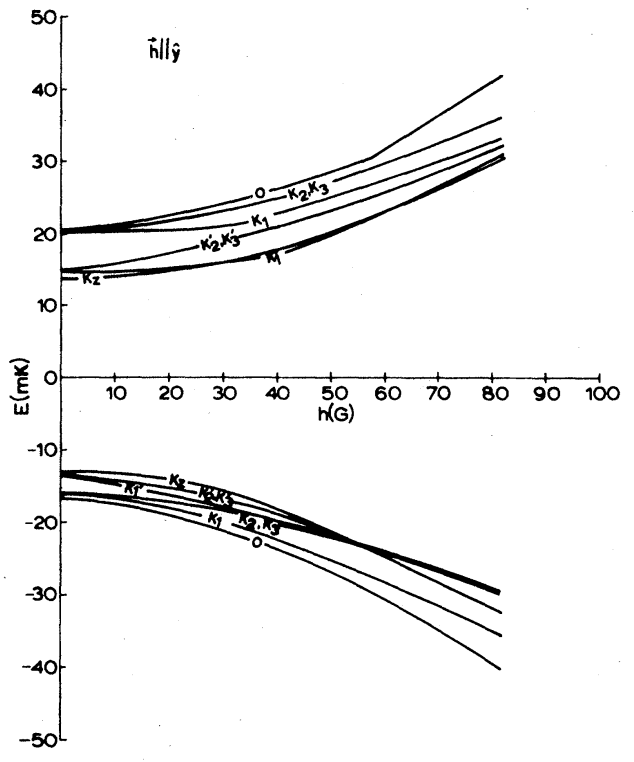

FIG. 4. Maximum and minimum energy for any $k$ representation versus magnetic field when the field is parallel to $y$ axis.

there, because of the calculation being in closed form, one can find exactly the field above which the state becomes metamagnetic. This field is $66.7 \mathrm{G} .^{12}$ Since the classical treatment does not involve lengthy calculations it is of interest to compare it with the quantum-mechanical one. For the $x$-direction the energy of the ground state below the critical field is by about $10 \%$ smaller than the quantum-mechanical case. The critical fields too differ by about $20 \%$. Beyond the critical field of the quantummechanical case the energies coincide. Thus the classical calculations can give only a first estimate of the groundstate energy and the critical field (see Fig. 5). For the $y$ direction, the estimate comes closer; see Fig. 6.

One of the quantities which can be easily measured experimentally is the susceptibility or equivalently the magnetization. Our calculations for the magnetic moment per cluster, i.e., for an octade of $\mathrm{Ce}$ ions, appear in Fig. 7. For the $x$ direction a clear phase transition appears at the

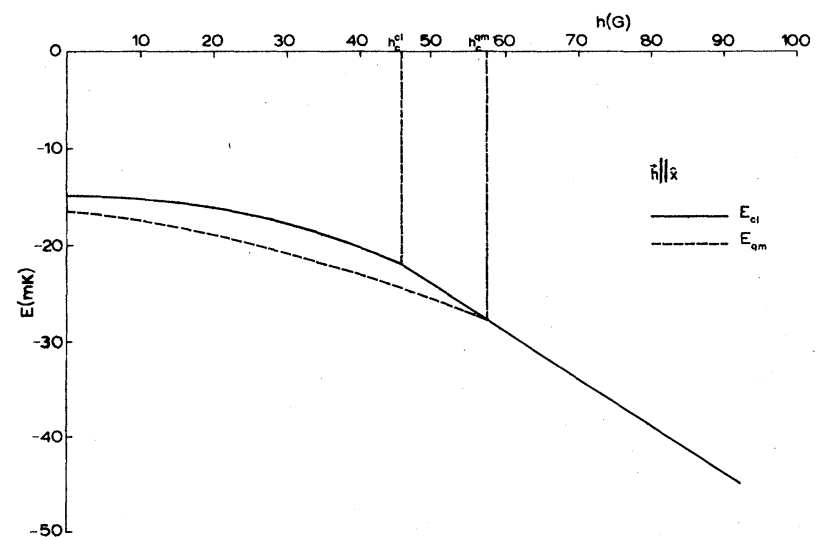

FIG. 5. Ground-state energy versus magnetic field parallel to $x$ axis. The solid line denotes the semiclassical treatment and the dashed line denotes the quantum-mechanical one. The values of the critical field is $h_{c}^{c 1}=45.8 \mathrm{G}$ and $h_{c}^{q m}=57.5 \mathrm{G}$, respectively.

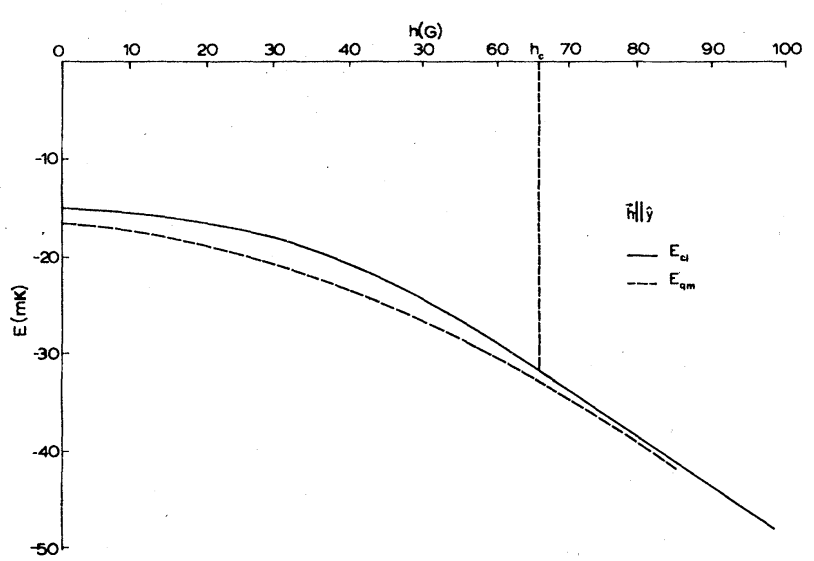

FIG. 6. Ground-state energy versus magnetic field parallel to $y$ axis. The solid line denotes the semiclassical treatment and the dashed line denotes the quantum-mechanical one. Critical field, $h_{c}^{c 1}=66.7 \mathrm{G}$, appears only to the semiclassical treatment.

critical field above which the spins turn abruptly toward the field direction. This is the case with the classical treatment also. ${ }^{1}$ However, in the classical case transverse components of the magnetization appear, i.e., magnetization normal to the applied external field (see Fig. 6, Ref. 1). Such anisotropy does not appear either in the quantum-mechanical approach or in the experiment. ${ }^{2,4}$ Thus, as far as magnetization is concerned, the classical case is inadequate as it gives qualitatively different results. The susceptibility for the classical case increases with the magnetic field (contrary to the quantum-mechanical treatment which is practically a straight line), in qualitative agreement with the experiment. ${ }^{2,4}$ When the field is along the $y$ direction the two treatments give about the same results.

As for the calculation of thermodynamic quantities it is necessary to know the density of states. We thought it useful to present our results for these curves, Figs. 1(a)-1(e). Since the spectrum is not continuous the number of states, $N(E)$, was averaged over energy spacings of $1 \mathrm{mK}$. The general feature is that as the magnetic field increases there is an energy spread of the states. Energy gaps appear in certain energy regions for zero external

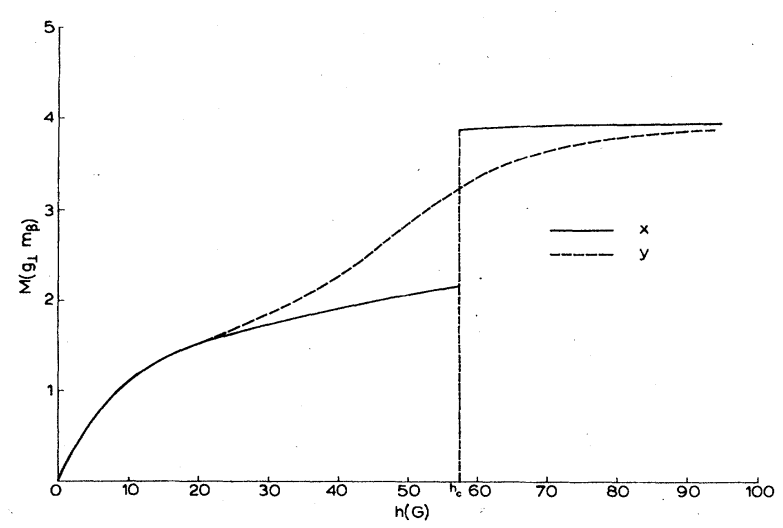

FIG. 7. Magnetic moment versus magnetic field. Solid and dashed lines denote the cases when the field is parallel to $x$ or $y$ axis. The critical field at $57.5 \mathrm{G}$ appears very unreadable in the first case. 
fields. The lowest such gap is in the millikelvin region of the transition temperature. ${ }^{2,3}$ Of course the real number of states of such a crystal is infinite and not just $2^{8}$ as presented here. It is of interest to know whether these results of density of states present the trend for the exact solution and whether these can be used to give thermodynamic quantities.

Hitherto our results were presented by neglecting the correction term [see Eq. (6)]. After this term is taken into account the change in energy is very small, ranging from 0 to $0.1 \mathrm{mK}$. Since the percentage of energy change is less than $0.6 \%$, the contribution of the correction factor is negligible. This justifies the approximation method used for finding the eigenstates of $H$.

\section{ACKNOWLEDGMENT}

Part of this work was performed at the "Demokritos" Nuclear Research Center while one of the authors was working for his Ph.D. thesis.
${ }^{1}$ A. K. Theophilou and S. Thanos, J. Chem. Phys. 72, 5649 (1980).

${ }^{2}$ R. A. Fisher, E. W. Hornung, G. E. Brodale, and W. E. Giaugue, J. Chem. Phys. 58, 5584 (1973).

${ }^{3}$ K. W. Mess, J. Lubbers, L. Niesen, and W. J. Huiskamp, Physica (Utrecht) 41, 260 (1969).

${ }^{4}$ D. J. Abeshouse, G. O. Zimmerman, D. R. Kelland, and E. Maxwell, Phys. Rev. Lett. 23, 308 (1969).

${ }^{5}$ Th. Niemeijer, Physica 57, 281 (1972).

6S. Thanos and A. K. Theophilou, Phys. Rev. B 181534 (1983).

${ }^{7}$ S. Thanos and A. K. Theophilou, Phys. Rev. B 28, 1544 (1983).

${ }^{8}$ P. H. E. Meijer and Th. Niemeijer, Phys. Rev. B 7, 1984 (1973).
${ }^{9}$ Th. Niemeijer and P. H. E. Meijer, Phys. Rev. B 10, 2962 (1974).

10P. H. E. Meijer and Th. Neimeijer, Phys. Rev. B 11, 2612 (1975).

11J. M. Luttinger and L. Tisza, Phys. Rev. 70, 954 (1946).

${ }^{12}$ A. Theophilou, Physica (Utrecht), 70, 599 (1973).

${ }^{13}$ M. Marrenga and Th. Niemeijer, Physica (Utrecht) 72, 624 (1974).

${ }^{14}$ H. W. Broughton and W. J. Mullin, Phys. Rev. B 6, 277 (1972).

${ }^{15}$ A. Zalkin, J. D. Forrester, and D. H. Templeton, J. Chem. Phys. 39, 2881 (1963). 